\title{
Do the Morningness-Eveningness questionnaire and Munich ChronoType questionnaire change after morning light treatment?
}

\author{
Helen J. Burgess ${ }^{1 *}$, Fumitaka Kikyo ${ }^{1}$, Zerbrina Valdespino-Hayden², Muneer Rizvydeen ${ }^{1}$, Momoko Kimura ${ }^{1}$, \\ Mark H. Pollack', Stevan E. Hobfoll1, Kumar B. Rajan³, Alyson K. Zalta ${ }^{1,2}$ and John W. Burns ${ }^{1}$
}

\begin{abstract}
The Morningness-Eveningness Questionnaire (MEQ) and Munich ChronoType Questionnaire (MCTQ) are sometimes used to estimate circadian timing. However, it remains unclear if they can reflect a change in circadian timing after a light treatment. In this study, 31 participants (25-68 years) completed both questionnaires before and after a 13-28 day morning light treatment. The dim light melatonin onset (DLMO), a physiological marker of circadian timing, was also assessed in a subsample of 16 participants. The DLMO phase advanced on average by 47 min $(p<0.001)$. The MEQ score increased by 1.8 points $(p=0.046)$. The MSFsc measure derived from the MCTQ advanced by $8.7 \mathrm{~min}(p=0.17)$. The shift towards morningness observed in both questionnaires correlated with the phase advance observed in the DLMO (MEQ $r=-0.46, p=0.036$; MSFsc $r=0.81, p<0.001$ ). Results suggest that these circadian questionnaires can change in response to a light treatment, indicating they can reflect underlying changes in circadian timing.
\end{abstract}

Trial registration: Clinicaltrials.gov NCT02373189 retrospectively registered 2/26/15; NCT03513848 retrospectively registered 5/2/18.

Keywords: Circadian, Human, Light, Melatonin, Sleep

\section{Introduction}

The dim light melatonin onset (DLMO) is the most reliable measure of central circadian timing in humans (Lewy et al. 1999; Klerman et al. 2002). The onset of the secretion of melatonin, which is tightly controlled by the central circadian clock (suprachiasmatic nucleus, $\mathrm{SCN}$ ) (Moore 1996), typically begins $2-3 \mathrm{~h}$ before habitual sleep onset (Burgess and Fogg 2008). The melatonin rhythm must be measured in dim light, as it is suppressed by light (Lewy et al. 1980). The DLMO can usually be obtained from saliva samples, collected every half-hour or hour, in the $6 \mathrm{~h}$ window before habitual sleep onset (Burgess and Fogg 2008). However, there are significant disadvantages in measuring the DLMO: it requires staff to assist in the collection and processing of

\footnotetext{
* Correspondence: Helen_」_Burgess@rush.edu

${ }^{1}$ Biological Rhythms Research Laboratory, Department of Behavioral Sciences, Rush University Medical Center, Chicago, IL, USA

Full list of author information is available at the end of the article
}

samples, significant participant effort, and the melatonin assay can be costly ( $\sim \$ 14$ per sample). For these reasons, there remains considerable interest in estimating circadian timing with questionnaires.

Two such questionnaires include the MorningnessEveningness Questionnaire (MEQ) (Horne and Ostberg 1976), and the Munich ChronoType Questionnaire (MCTQ) (Roenneberg et al. 2003). As we reviewed previously (Kantermann et al. 2015), the MEQ includes 19 questions that ask people to consider their "feeling best" rhythms and indicate preferred clock time blocks for sleep and engagement in various hypothetical activities (e.g. physical exercise, tests, work), in addition to assessing morning alertness, morning appetite, evening tiredness and alarm clock dependency. MEQ scores can range from 16 to 86 , with lower scores indicating

(c) The Author(s). 2018 Open Access This article is distributed under the terms of the Creative Commons Attribution 4.0 International License (http://creativecommons.org/licenses/by/4.0/), which permits unrestricted use, distribution, and reproduction in any medium, provided you give appropriate credit to the original author(s) and the source, provide a link to the Creative Commons license, and indicate if changes were made. The Creative Commons Public Domain Dedication waiver (http://creativecommons.org/publicdomain/zero/1.0/) applies to the data made available in this article, unless otherwise stated. 
eveningness or later circadian timing, and higher scores indicating morningness or earlier circadian timing. By contrast, the MCTQ focuses primarily on sleep timing and via 14 questions, assesses the regularity of one's work schedule, number of workdays per week, sleep timing on workdays and work-free days, and alarm clock use on workdays and work-free days. Circadian timing is estimated as the midpoint of sleep on work-free days minus half of the difference between sleep duration on work-free days and average sleep duration of the week to control for sleep debt (midpoint of sleep on work-free days, sleep corrected, referred to from here on as MSFsc). Importantly, the MSFsc should only be considered valid when individuals report not using an alarm clock on work-free days (Roenneberg et al. 2012). We and others have reported that the estimates of circadian timing generated from these questionnaires, do indeed correlate significantly with the DLMO (MEQ and DLMO rs $=-0.40$ to -0.70 ; MSFsc and DLMO rs $=0.54$ to 0.68; (Kitamura et al. 2014, Kantermann et al. 2015). A related, but to date unanswered, question is whether these questionnaires are sensitive enough to reflect an underlying change in circadian timing following a light treatment. If they do, this would further support their use as potential estimators of circadian timing when direct measurement of the DLMO is not feasible. Thus, the objective of this study was to examine these circadian questionnaires before and after a bright light treatment.

\section{Materials and methods}

Our sample consisted of 31 participants (23 males, 8 females, mean age $45.9 \pm 13.7$ years, mean BMI $30.3 \pm 6.0 \mathrm{~kg} / \mathrm{m}^{2}, 45 \%$ Non-Hispanic African American, 32\% Non-Hispanic White, 19\% Hispanic White, 3\% other) who were recruited from internet advertising (e.g. craigslist.com). The majority was not working (68\% not working, $26 \%$ part-time workers, $6 \%$ full-time workers), none had engaged in shift work in at least the past month, and all passed a urine drug test and alcohol breathalyzer test. The sample was derived from two separate clinical trials that tested the mood altering effects of a self-administered morning bright light treatment administered at home for 13-28 days. In both studies, the $1 \mathrm{~h}$ light treatment was scheduled to start each morning at the subject's average wake time (derived from a baseline week of wrist actigraphy collected just prior to the start of the light treatment), or up to $1 \mathrm{~h}$ earlier to accommodate morning social responsibilities (e.g. work, child care). In the first trial, 23 U.S. military veterans with chronic low back pain received morning bright light from two broad-spectrum white light boxes that research staff set up in their homes $(33 \times 18 \times$ $55 \mathrm{~cm}$, EnergyLight HF3318/60, Philips, Inc., generated > $3000 \mathrm{~lx}$ at subjects' eyes). The light treatment was scheduled for 13 consecutive mornings (NCT02373189 on clinicaltrials.gov). Light readings from photosensors attached to the light boxes were checked against light readings on each subject's wrist monitor (Actiwatch Spectrum, Philips, Inc) to monitor adherence to the light treatment. In the second trial, 8 subjects with probable post-traumatic stress disorder (Post-Traumatic Stress Disorder Checklist for DSM-5 score > 33, (Weathers et al. 2013, Bovin et al. 2016)) received morning light from a wearable light device, the Re-timer $(20 \times$ $14 \times 5.5 \mathrm{~cm}$, Re-time, Inc., Australia, generated $\sim 500 \mathrm{~lx}$ at subjects' eyes, with peak wavelength of $\sim 500 \mathrm{~nm}$ ). The Re-timer was individually adjusted to each subject to optimize the light treatment, which was scheduled for 28 consecutive mornings (NCT03513848 on clinicaltrials.gov). Light and activity readings from a monitor (Actiwatch Spectrum, Philips, Inc) attached to the inside of the Re-timer were used to monitor adherence to the light treatment. All subjects included in this report received the morning light treatment on at least $80 \%$ of the assigned mornings.

The MEQ and MCTQ were measured at the end of the baseline week, which was also the day before the start of the light treatment. They were then re-measured on the day of the last light treatment, after the light treatment had concluded. In the veteran trial, a validated home saliva collection kit (Burgess et al. 2015; Burgess et al. 2016) was used to assess the dim light melatonin onset (DLMO) at the same pre and post-treatment time points. Saliva samples were collected every half hour for $6 \mathrm{~h}$ in $\operatorname{dim}$ light $(<50 \mathrm{~lx})$, starting $6 \mathrm{~h}$ before average sleep onset time. All subjects refrained from caffeine and alcohol in at least the $24 \mathrm{~h}$ before saliva collection, and refrained from non-steroidal anti-inflammatory drugs for at least $72 \mathrm{~h}$ before saliva collection. No participants were taking beta-blockers or exogenous melatonin. Melatonin levels were derived from the saliva samples by Solidphase Inc. (Portland, ME), with a direct radioimmunoassay using standard Buhlmann kits with assay sensitivity of $0.5 \mathrm{pg} / \mathrm{ml}$, intra and interassay $\mathrm{CV}<7.5 \%$ at $3 \mathrm{pg} / \mathrm{ml}$. The DLMO was calculated as the clock time (with linear interpolation) when the melatonin concentration exceeded the mean of 3 low consecutive daytime values plus twice the standard deviation of these points (Voultsios et al. 1997). This low threshold more closely tracks the initial rise of melatonin, when the SCN triggers the release of melatonin from the pineal gland (Molina and Burgess 2011). The DLMOs for seven veterans were not valid due to low levels of melatonin $(<5 \mathrm{pg} / \mathrm{ml})$ or erratic melatonin profiles. The DLMO was not assessed in the PTSD trial. The Rush University Medical Center Institutional Review Board approved both study protocols, which followed the principles of the Declaration of Helsinki. All subjects gave written informed consent prior to participation. The changes in DLMO, MEQ and MSFsc (derived from the MCTQ) were analyzed with a paired samples t-test. As morning light is well recognized to cause circadian phase 
advances, an increase in morningness was predicted, and a one-tailed $p$-value of $<0.05$ was used to determine statistical significance.

\section{Results}

The variables were normally distributed. The changes in the DLMO, MEQ score and MSFsc from pre- to postlight treatment are shown in Fig. 1. On average, the DLMO significantly phase advanced by $47 \mathrm{~min}(p<0.001$, $n=16, \mathrm{~d}=1.49)$. The MEQ score significantly increased by 1.8 points ( $p=0.046, n=31, \mathrm{~d}=0.32$ ), reflecting more morningness after the morning light treatment. The MSFsc advanced by $8.7 \mathrm{~min}$, but this was not statistically significant $(p=0.17, \mathrm{n}=31, \mathrm{~d}=0.17)$. Nine subjects reported using alarm clocks on their non-work days. With those subjects removed the advance in MSFsc increased to $14.4 \mathrm{~min}$, but this change was still not significant $(p=0.12, n=22, \mathrm{~d}=0.25)$. Overall, the phase advance in the DLMO correlated with an increase in morningness on both questionnaires (MEQ $r=-0.46, p=0.036$; MSFsc $r=0.81, p<0.001, \mathrm{n}=16)$.

\section{Discussion}

These results indicate that the MEQ and MCTQ questionnaires can reflect an increase in morningness following a morning light treatment. The MEQ score increased significantly, reflecting more morningness, and also correlated with the phase advance in the DLMO. The MSFsc derived from the MCTQ did not significantly change with the light treatment, but the increase in morningness did correlate significantly with the degree of circadian phase advance observed in the DLMO. Thus these results further support the use of these circadian questionnaires as potential estimators of circadian timing when direct measurement of the DLMO is not feasible. Contrary to other sleep questionnaires, the MEQ and MCTQ (at least the earlier version we used in this study) do not have any time frame in their instructions for completion, such as "in the past week" which is used in the Insomnia Severity Index (Bastien et al. 2001) and "during the past month only" which is used in the Pittsburgh Sleep Quality Index (Buysse et al. 1989). This lack of time reference may have reduced the ability to detect differences in the MEQ score and MSFsc, with assessments only 2-4 weeks apart. The greater sensitivity of the MEQ to the morning light treatment may be due to the questions in the MEQ assessing a broader range of activities beyond sleep, including for example people's "feeling best" rhythms and preferred clock times for engagement in various hypothetical activities (e.g. physical exercise, tests, work), in addition to assessing morning alertness, morning appetite, evening tiredness and alarm clock dependency. By contrast, the MCTQ focuses primarily on sleep timing and alarm clock use, and responses may be more constrained by perceived societal
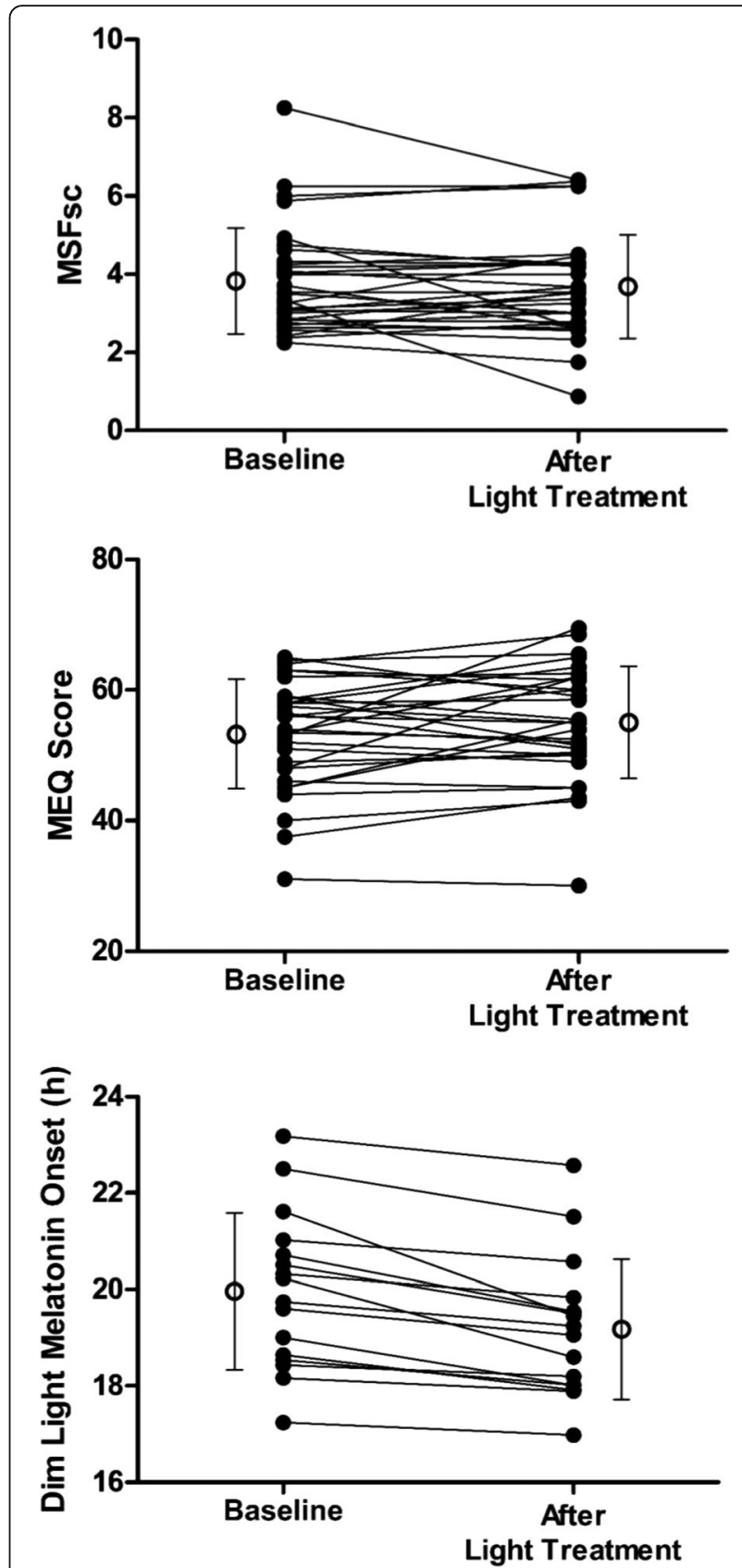

Fig. 1 The changes in MSFsc, MEQ score, and DLMO observed in each individual subject before and after a 13 or 28 day morning light treatment. The results for an individual subject are connected by a line. The mean and standard deviation at each time point is also shown

norms surrounding usual sleep times. Additionally, even though the majority of our sample was not working, almost a third of subjects reported using an alarm clock on their work-free days, which may reflect other non-work social responsibilities. It remains unclear if these subjects were reporting the setting of an alarm on these days, or the actual waking to an alarm clock on those days. Further, use of an alarm clock on work-free days technically 
invalidates the MSFsc (Roenneberg et al. 2012), indicating the higher potential for missing data when using the MCTQ than when using the MEQ.

There are several limitations to our study. We were not able to assess DLMO in all subjects, and therefore cannot verify a significant circadian phase advance occurred in the entire sample. Nonetheless, the subsample of 16 subjects revealed a statistically significant phase advance of $\sim 50 \mathrm{~min}$ in response to the morning bright light, which is remarkably consistent with the phase advance seen in the phase response curve to $1 \mathrm{~h}$ of light, when administered at average wake time $(\sim 14 \mathrm{~h}$ after the DLMO, Figure 3 in (St. Hilaire et al. 2012)). Additionally, the Re-timer has been shown to elicit phase shifts in the DLMO (Lovato and Lack 2016). Given that objectively measured adherence to the light treatment in both trials was reasonable, it is likely that on average the entire sample phase advanced in response to the morning light treatment. Indeed the increase of $\sim 2$ points in the MEQ observed in the full sample, was similar to that observed in the subsample in which the DLMO was measured. We also note that we have no control group, and thus no measure of the natural fluctuations in these circadian questionnaires over time. We also recognize that our sample size was small, and therefore our analyses were underpowered. We encourage other researchers assessing the circadian effects of light treatment to consider administering these circadian questionnaires both before and after a light treatment to further explore the sensitivity of these questionnaires in larger samples. To our knowledge this is rarely done in light treatment studies. Future work should also examine these relationships in larger non-clinical samples (Di Milia et al. 2013), as our sample is not necessarily representative of the general population and was largely male. It would also be interesting to determine if circadian phase shifts due to other non-photic stimuli, such as exogenous melatonin, could also significantly shift the scores derived from these circadian questionnaires.

\section{Abbreviations \\ BMI: Body mass index; DLMO: Dim light melatonin onset; MCTQ: Munich ChronoType Questionnaire; MEQ: Morningness-Eveningness Questionnaire; MSFsc: Midpoint of sleep on work-free days sleep corrected; PTSD: Post- traumatic stress disorder; $\mathrm{SCN}$ : Suprachiasmatic nucleus}

\section{Acknowledgements}

We thank Karyna Bravo, Morgan Corich, Joshua Dein, Aahad Kahn, Catherine Keefner, Mary Kennedy, Athanasios Kondilis, Othon Nunez-Montelongo, Daria Orlowska, Philip Sanchez, Monica Thomas, Marie Vallido, Amanda Vatinno, and Denise Zou for their assistance with data collection. Research reported in this publication was supported by the National Center for Complementary and Integrative Health of the National Institutes of Health under Award Number R34AT008347, and by internal funds provided by the Department of Psychiatry at Rush University Medical Center. Alyson Zalta's contribution was supported by a training grant from the National Institute of Mental Health (K23 MH103394). The content is solely the responsibility of the authors and does not necessarily represent the official views of the National Institutes of Health.

\section{Competing interests}

Dr. Burgess is a consultant for Natrol, LLC. Dr. Pollack reports personal fees from Aptinyx, Bracket Global, Palo Alto Health Sciences, grants from Janssen, outside the submitted work. In addition, Dr. Pollack has a patent SIGHA, SAFER interviews with royalties paid and Equity in Argus, Doyen Medical, Mensante Corporation, Mindsite, Targia Pharmaceuticals. All other authors report no conflicts of interest.

\section{Authors' contributions}

All authors read and approved the final manuscript.

\section{Publisher's Note}

Springer Nature remains neutral with regard to jurisdictional claims in published maps and institutional affiliations.

\section{Author details}

${ }^{1}$ Biological Rhythms Research Laboratory, Department of Behavioral Sciences, Rush University Medical Center, Chicago, IL, USA. ${ }^{2}$ Department of Psychiatry, Rush University Medical Center, Chicago, IL, USA. ${ }^{3}$ Department of Internal Medicine, Rush University Medical Center, Chicago, IL, USA.

Received: 12 July 2018 Accepted: 22 August 2018

Published online: 16 September 2018

\section{References}

Bastien CH, Vallieres A, Morin CM. Validation of the insomnia severity index as an outcome measure for insomnia research. Sleep Med. 2001;2(4):297-307.

Bovin MJ, Marx BP, Weathers FW, Gallagher MW, Rodriguez P, Schnurr PP, Keane TM. Psychometric properties of the PTSD checklist for diagnostic and statistical manual of mental disorders-fifth edition (PCL-5) in veterans. Psychol Assess. 2016;28(11):1379-91.

Burgess HJ, Fogg LF. Individual differences in the amount and timing of salivary melatonin secretion. PLoS One. 2008;3(8):e3055.

Burgess HJ, Park M, Wyatt JK, Fogg LF. Home dim light melatonin onsets with measures of compliance in delayed sleep phase disorder. J Sleep Res. 2016; 25(3):314-7.

Burgess HJ, Wyatt JK, Park M, Fogg LF. Home circadian phase assessments with measures of compliance yield accurate dim light melatonin onsets. Sleep. 2015;38(6):889-97.

Buysse DJ, Reynolds CF, Monk TH, Berman SR, Kupfer DJ. The Pittsburgh sleep quality index: a new instrument for psychiatric practice and research. Psychiatry Res. 1989;28:193-213.

Di Milia L, Adan A, Natale V, Randler C. Reviewing the psychometric properties of contemporary circadian typology measures. Chronobiol Int. 2013;30(10): $1261-71$.

Horne J, Ostberg O. A self-assessment questionnaire to determine morningnesseveningness in human circadian rhythms. Int J Chronobiol. 1976:4:97-110.

Kantermann T, Sung H, Burgess HJ. Comparing the Morningness-Eveningness questionnaire and Munich ChronoType questionnaire to the dim light melatonin onset. J Biol Rhythm. 2015;30(5):449-53.

Kitamura S, Hida A, Aritake S, Higuchi S, Enomoto M, Kato M, Vetter C, Roenneberg T, Mishima K. Validity of the Japanese version of the Munich ChronoType questionnaire. Chronobiol Int. 2014;31(7):845-50.

Klerman EB, Gershengorn HB, Duffy JF, Kronauer RE. Comparisons of the variability of three markers of the human circadian pacemaker. J Biol Rhythm. 2002;17(2):181-93.

Lewy AJ, Cutler NL, Sack RL. The endogenous melatonin profile as a marker of circadian phase position. J Biol Rhythm. 1999;14(3):227-36.

Lewy AJ, Wehr TA, Goodwin FK, Newsome DA, Markey SP. Light suppresses melatonin secretion in humans. Science. 1980;210(4475):1267-9.

Lovato N, Lack L. Circadian phase delay using the newly developed re-timer portable light device. Sleep Biol Rhythms. 2016;14:157-64.

Molina TA, Burgess HJ. Calculating the dim light melatonin onset: the impact of threshold and sampling rate. Chronobiol Int. 2011;28(8):714-8.

Moore RY. Neural control of the pineal gland. Behavioral Brain Research. 1996;73: $125-30$.

Roenneberg T, Allebrandt KV, Merrow M, Vetter C. Social jetlag and obesity. Curr Biol. 2012;22(10):939-43.

Roenneberg T, Wirz-Justice A, Merrow M. Life between clocks: daily temporal patterns of human chronotypes. J Biol Rhythm. 2003;18(1):80-90. 
St Hilaire MA, Gooley JJ, Khalsa SB, Kronauer RE, Czeisler CA, Lockley SW. Human phase response curve to a $1 \mathrm{~h}$ pulse of bright white light. J Physiol. 2012; 590(Pt 13):3035-45.

Voultsios A, Kennaway DJ, Dawson D. Salivary melatonin as a circadian phase marker: validation and comparison to plasma melatonin. J Biol Rhythm. 1997; 12(5):457-66.

Weathers FW, Litz BT, Keane TM, Palmieri PA, Marx BP, Schnurr PP. 2013. The PTSD checklist for DSM-5 (PCL-5). Scale available from the National Center for PTSD at www.ptsd.va.gov.

Ready to submit your research? Choose BMC and benefit from:

- fast, convenient online submission

- thorough peer review by experienced researchers in your field

- rapid publication on acceptance

- support for research data, including large and complex data types

- gold Open Access which fosters wider collaboration and increased citations

- maximum visibility for your research: over $100 \mathrm{M}$ website views per year

At $\mathrm{BMC}$, research is always in progress.

Learn more biomedcentral.com/submissions 